\title{
Safety and Feasibility of the OmniPod Hybrid Closed-Loop System in Adult, Adolescent, and Pediatric Patients with Type 1 Diabetes Using a Personalized Model Predictive Control Algorithm
}

\author{
Bruce A. Buckingham, MD, Gregory P. Forlenza, MD, Jordan E. Pinsker, MD, \\ Mark P. Christiansen, MD, R. Paul Wadwa, MD, Jennifer Schneider, MD, \\ Thomas A. Peyser, PhD, Eyal Dassau, PhD, Joon Bok Lee, $\mathrm{PhD}^{7}$, Jason $\mathrm{O}^{\prime}$ Connor, BS, \\ Jennifer E. Layne, $\mathrm{PhD}^{7}$ and Trang T. Ly, MBBS, FRACP, $\mathrm{PhD}^{7}$
}

\begin{abstract}
Background: The safety and feasibility of the OmniPod personalized model predictive control (MPC) algorithm in adult, adolescent, and pediatric patients with type 1 diabetes were investigated.

Methods: This multicenter, observational trial included a 1-week outpatient sensor-augmented pump open-loop phase and a 36-h inpatient hybrid closed-loop (HCL) phase with announced meals ranging from 30 to $90 \mathrm{~g}$ of carbohydrates and limited physical activity. Patients aged 6-65 years with HbA1c between $6.0 \%$ and $10.0 \%$ were eligible. The investigational system included a modified version of OmniPod, the Dexcom G4 505 Share ${ }^{\circledR}$ AP System, and the personalized MPC algorithm running on a tablet computer. Primary endpoints included sensor glucose percentage of time in hypoglycemia $<70 \mathrm{mg} / \mathrm{dL}$ and hyperglycemia $>250 \mathrm{mg} / \mathrm{dL}$. Additional glycemic targets were assessed.

Results: The percentage of time $<70 \mathrm{mg} / \mathrm{dL}$ during the $36-\mathrm{h} \mathrm{HCL}$ phase was mean (standard deviation): 0.7 (1.7) in adults receiving 80\% meal bolus $(n=24)$, and $0.7(1.2)$ in adults $(n=10), 2.0(2.4)$ in adolescents $(n=12)$, and $2.0(2.6)$ in pediatrics $(n=12)$ receiving $100 \%$ meal bolus. The overall hypoglycemia rate was 0.49 events $/ 24 \mathrm{~h}$. The percentage of time $>250 \mathrm{mg} / \mathrm{dL}$ was 8.0 (7.5), $3.6(3.7), 4.9$ (6.3), and 6.7 (5.6) in the study groups, respectively. Percentage of time in the target range of $70-180 \mathrm{mg} / \mathrm{dL}$ was 69.5 (14.4), 73.0 (15.0), 72.6 (15.5), and 70.1 (12.3), respectively.

Conclusions: The OmniPod personalized MPC algorithm performed well and was safe during day and night use in adult, adolescent, and pediatric patients with type 1 diabetes. Longer term studies will assess the safety and performance of the algorithm under free living conditions with extended use.
\end{abstract}

Keywords: Artificial pancreas, Automated insulin delivery, Closed loop, Hybrid closed loop, OmniPod, Tubeless insulin pump.

\footnotetext{
${ }^{1}$ Division of Endocrinology, Department of Pediatrics, Stanford School of Medicine, Stanford, Palo Alto, California.

${ }^{2}$ Barbara Davis Center for Diabetes, University of Colorado School of Medicine, Aurora, Colorado.

${ }^{3}$ Sansum Diabetes Research Institute, Santa Barbara, California.

${ }^{4}$ Diablo Clinical Research, Walnut Creek, California.

${ }^{5}$ ModeAGC LLC, Palo Alto, California.

${ }^{6}$ Harvard John A. Paulson School of Engineering and Applied Sciences, Harvard University, Cambridge, Massachusetts.

${ }^{7}$ Insulet Corporation, Billerica, Massachusetts.

Data included in this article were presented, in part, at the 10th International Conference on Advanced Technologies and Treatments for Diabetes, February 15-18, 2017, in Paris, France, and at the 77th Scientific Sessions of the American Diabetes Association, June 9-13, 2017, in San Diego, CA.

(c) Bruce A. Buckingham, et al., 2018; Published by Mary Ann Liebert, Inc. This Open Access article is distributed under the terms of the Creative Commons Attribution Noncommercial License (http://creativecommons.org/licenses/by-nc/4.0/) which permits any noncommercial use, distribution, and reproduction in any medium, provided the original author(s) and the source are credited.
} 


\section{Introduction}

C LOSED-LOOP ARTIFICIAL PANCREAS (AP) systems have been in development for several years, all with the goal of reducing the burden of diabetes through automated insulin delivery. Advances in microelectronics and glucose sensor technology and the U.S. Food and Drug Administration's (FDA) decision to make the development of closed-loop systems a priority have resulted in rapid advances in this field. ${ }^{1,2}$ The first commercial closed-loop device was recently approved by the FDA in September $2016,{ }^{3}$ following a 3 month clinical study of 124 subjects aged $14-75$ years that demonstrated few serious or device-related adverse events. ${ }^{4,5}$ A recent meta-analysis of randomized controlled trials has demonstrated that AP systems consistently improve glycemic control across age groups compared to continuous subcutaneous insulin infusion (CSII) with blinded continuous glucose monitoring (CGM) or with sensor-augmented pumps (SAP). ${ }^{6}$ In the outpatient setting, the use of AP systems has resulted in a $12 \%$ increased time in range $(70-180 \mathrm{mg} / \mathrm{dL})$ and a $50 \%$ reduction in the time spent in hypoglycemia. ${ }^{6}$

There are several AP systems currently under development in both academic and commercial endeavors. Each system offers unique features in the configuration of pumps, glucose sensors, algorithms, functionality, user interface, and data management. Systems are also differentiated by having single- or dual-hormone delivery. ${ }^{1}$

Early feasibility studies of the OmniPod ${ }^{\circledR}$ Horizon Automated Glucose Control system, a single-hormone hybrid closed-loop (HCL) system, are currently underway. The commercial system is intended to be a fully on-body device with a personalized Model Predictive Control (MPC) algorithm embedded in the OmniPod patch pump (Pod) communicating directly with the latest Dexcom CGM technology. A handheld device will transmit commands for insulin meal boluses, algorithm parameter adjustments such as change in target glucose and insulin delivery parameters, as well as allowing connection to a secure cloud server for data management.

The objectives of this feasibility study were to evaluate the safety and feasibility of the OmniPod personalized MPC algorithm in adult, adolescent, and pediatric patients with type 1 diabetes in a clinical research center setting.

\section{Materials and Methods}

This single-arm, observational clinical trial study was conducted at four sites and consisted of a 1-week, outpatient open-loop phase using SAP and a 36-h inpatient HCL phase using the OmniPod HCL investigational device.

During the open-loop phase, subjects managed their diabetes at home as per their usual routine using their personal insulin pump and the study CGM (Dexcom G4 505 Share ${ }^{\circledR}$ AP System). Investigators analyzed $\sim 7$ days of open-loop data and adjusted pump settings for basal rate, insulin to carbohydrate ratio, and correction factor as needed, based on their clinical judgment, for safety before proceeding to the HCL phase.

The HCL phase began before breakfast on day 1 and concluded $\sim 4 \mathrm{~h}$ after lunch on day 2 . The HCL phase included five meals with 30 to $90 \mathrm{~g}$ carbohydrate per meal, one overnight period, and limited physical activity. Subjects se- lected meals from a variety of food options with identical meals given for breakfast and lunch on days 1 and 2. During the HCL phase, subjects were closely monitored by study personnel. Hypoglycemia safety actions included treatment with 12 to $16 \mathrm{~g}$ of fast-acting carbohydrates for CGM values $<70 \mathrm{mg} / \mathrm{dL}$ with a confirmatory fingerstick. A follow-up fingerstick was performed $15 \mathrm{~min}$ post-treatment to assess resolution, defined as blood glucose $\geq 70 \mathrm{mg} / \mathrm{dL}$, or the need for additional treatment as per clinical standards. Fast-acting carbohydrates were also available to any subject who was symptomatic or requested treatment. Hyperglycemia treatment, a correction bolus target of $150 \mathrm{mg} / \mathrm{dL}$, was initiated for CGM values of $\geq 300 \mathrm{mg} / \mathrm{dL}$ confirmed by fingerstick and ketones $\leq 1.0 \mathrm{mmol} / \mathrm{L}$. HCL stopping criteria included blood glucose $\geq 300 \mathrm{mg} / \mathrm{dL}$ with ketones $\geq 3.0 \mathrm{mmol} / \mathrm{L}$, subject unable to take oral carbohydrates, subject request, loss of consciousness, or seizure.

Pods were changed at the discretion of the investigator in cases of ketones $>1.0 \mathrm{mmol} / \mathrm{L}$ or if there was a suspected infusion site failure. Subjects were discharged on day 2 when deemed safe by the investigators.

Two meal bolus protocols were used in the study. As this was the first human clinical study of the modified MPC algorithm, a conservative approach of $80 \%$ meal bolus was administered to the initial 24 adult subjects. The modified algorithm was determined to be safe and all subjects subsequently enrolled in the study received $100 \%$ meal bolus.

\section{Subjects}

Enrollment criteria include patients 6 to 65 years of age, type 1 diabetes for $\geq 1$ year, $\mathrm{HbA} 1 \mathrm{c}$ value $>6 \%$ and $<10 \%$ within the past 6 months, use of any insulin pump for $\geq 6$ months, and total daily dose of insulin $>0.4 \mathrm{U} / \mathrm{kg}$. Patients with CGM experience were not excluded. Patients with $\geq 1$ episode of severe hypoglycemia or diabetic ketoacidosis requiring an emergency room visit or hospitalization within the past 6 months or hypoglycemic unawareness assessed by the Clarke Questionnaire ${ }^{7}$ were excluded. The study obtained institutional review board approvals and written informed consent from adults and the parents or guardians of minors. Written assent was obtained from all minors over $\geq 8$ years of age.

\section{Investigational device}

System architecture. The investigational system used in this study consisted of a modified version of the Pod, a modified Personal Diabetes Manager (PDM), the Dexcom G4 505 Share AP System, and the personalized MPC algorithm running on a Windows 10 tablet configured with the portable AP System developed at the University of California at Santa Barbara. ${ }^{8}$ Operationally, the Dexcom CGM receiver communicated with the portable AP System (tablet) via a wired USB connection. The portable AP System transmitted insulin dosing commands to the PDM via Bluetooth Low Energy relay. The PDM subsequently controlled insulin delivery by the Pod via a radiofrequency signal.

The user interface on the tablet clearly indicated to clinical study staff whether the system was in HCL mode or in openloop mode. The tablet was used to start each Pod, initiate HCL, display CGM and insulin delivery data, and for meal bolus delivery and open-loop control, if needed. 
Personalized MPC algorithm. The OmniPod personalized MPC algorithm is a specific implementation of the MPC algorithm that was originally developed at the University of California at Santa Barbara by Doyle, Dassau, and colleagues $^{9,10}$ The algorithm has undergone years of extensive study, with further enhancements made before its implementation in the present study. The OmniPod personalized MPC algorithm insulin-dosing decisions are made every $5 \mathrm{~min}$ based on CGM values to minimize the deviation between predicted blood glucose over a 60 -min horizon and the target set point. Inputs to the algorithm include the subject-specific basal rate profile and total daily insulin dose. The algorithm incorporates an asymmetric cost function as described by Gondhalekar et al. to minimize the occurrence of hypoglycemia without compromising overall glycemic control. ${ }^{11}$ A dynamic insulin-on-board constraint is dependent on the subject-specific insulin sensitivity factor to ensure that insulin delivery rates are limited for safety purposes. The algorithm set point for this study was a sensor glucose of $120 \mathrm{mg} / \mathrm{dL}$.

\section{Outcomes}

Primary endpoints included safety parameters of percentage of time the sensor glucose was in a hypoglycemic range defined as $<70 \mathrm{mg} / \mathrm{dL}$ and percentage time in a hyperglycemic range defined as $\geq 250 \mathrm{mg} / \mathrm{dL}$ during the HCL phase. Secondary endpoints included sensor mean glucose, percentage time $\leq 50, \leq 60,70$ to 140,70 to $180, \geq 180, \geq 300 \mathrm{mg} /$ $\mathrm{dL}$, standard deviation, and coefficient of variation (CV) of CGM values. ${ }^{12}$

\section{Statistical analyses}

As the endpoint for the study was safety, sample size was not determined. Prespecified descriptive statistical analyses were performed for all subjects who entered the study $(n=58)$. Results were summarized for the 36-h HCL study period (overall) and the overnight period defined as 23:00 to 07:00. The endpoints were summarized based on a modified intention-to-treat analysis that included subjects who entered the HCL phase of the study. Results for adult subjects who received $80 \%$ meal bolus were summarized separately from adults who received $100 \%$ meal bolus. Statistical analyses were performed using SAS ${ }^{\circledR} 9.3$ (SAS Institute, Cary, NC).

\section{Results}

The characteristics of the 58 patients (adults $n=34$, age $\geq 18$ years; adolescents $n=12,12$ to $<18$ years; pediatrics $n=12,6$ to $<12$ years) are reported in Table 1 . In the openloop phase, 41 (70\%) subjects had adjustments to their basal rate with increases made for 26 subjects and decreases made for 15 subjects. Changes were made to the insulin to carbohydrate ratios for 25 subjects $(43 \%)$ with increases (decreased insulin delivery) in 4 subjects and decreases (increased insulin delivery) in 21 subjects. Correction factors were adjusted for $16(28 \%)$ subjects with increases (decreased insulin delivery) in 6 subjects and decreases (increased insulin delivery) in 10 subjects. Glycemic measures for the open-loop phase are reported in Table 2.

\section{Glycemic outcomes}

Glycemic outcomes for the 36-h HCL phase overall and overnight are presented in Tables 3 and 4 . The percentage of time in the hypoglycemic range $<70 \mathrm{mg} / \mathrm{dL}$ for sensor glucose during the HCL phase overall was mean (standard deviation [SD]): $0.7(1.7)$ in adults receiving $80 \%$ meal bolus $(n=24)$, and 0.7 (1.2) in adults $(n=10), 2.0(2.4)$ in adolescents $(n=12)$, and $2.0(2.6)$ in pediatrics $(n=12)$ receiving $100 \%$ meal bolus.

The percentage of time in the hyperglycemic range $>250 \mathrm{mg} / \mathrm{dL}$ during HCL was 8.0 (7.5), 3.6 (3.7), 4.9 (6.3), and 6.7 (5.6) in adults with $80 \%$ meal bolus, and in adults, adolescents, and pediatrics with $100 \%$ meal bolus, respectively. The percentage of time in target range of 70 to $180 \mathrm{mg} /$ $\mathrm{dL}$ was 69.5 (14.4), 73.0 (15.0), 72.6 (15.5), and 70.1 (12.3) in the study groups, respectively. During the overnight period, the percentage time in target range of 70 to $180 \mathrm{mg} / \mathrm{dL}$ was 87.8 (17.4), 90.8 (15.8), 84.7 (25.1), and 86.7 (17.7), respectively. Mean sensor glucose across age groups ranged from 153 (22) to 157 (20) overall and from 139 (21) to 149 (26) $\mathrm{mg} / \mathrm{dL}$ during the overnight period with $100 \%$ meal bolus. Sensor glucose profiles during the open-loop and HCL phases for each study cohort are provided in Supplementary Figures S1-S4 (Supplementary Data are available at http:// online.liebertpub.com/doi/suppl/10.1089/dia.2017.0346).

\section{Percentage time in closed loop}

Overall, the mean time in closed loop was $99.1 \%$ (range: $89.4 \%-100 \%)$. There were seven suspected infusion site

Table 1. Characteristics of the Study Population

\begin{tabular}{|c|c|c|c|c|}
\hline Characteristic & Adults $^{\mathrm{a}}(\mathrm{n}=24)$ & Adults $(\mathrm{n}=10)$ & Adolescents $(\mathrm{n}=12)$ & Pediatrics $(\mathrm{n}=12)$ \\
\hline Age, year & $37.1(14.7)$ & $41.7(18.1)$ & $14.6(1.5)$ & $9.5(1.7)$ \\
\hline Female, $\%$ & 67 & 90 & 50 & 42 \\
\hline Diabetes duration, year & $23.0(14.3)$ & $23.7(15.3)$ & $4.6(3.3)$ & $4.6(2.2)$ \\
\hline Insulin pump use duration, year & $12.8(7.0)$ & $12.6(8.0)$ & $3.2(2.6)$ & $3.6(2.1)$ \\
\hline Insulin dose open loop $[\mathrm{U} /(\mathrm{kg} \cdot \mathrm{d})]^{\mathrm{b}}$ & $0.61(0.21)$ & $0.53(0.24)$ & $0.83(0.26)$ & $0.79(0.12)$ \\
\hline Insulin dose $24-\mathrm{h} \mathrm{HCL}[\mathrm{U} /(\mathrm{kg} \cdot \mathrm{d})]^{\mathrm{c}}$ & $0.60(0.30)$ & $0.48(0.14)$ & $0.83(0.19)$ & $0.85(0.16)$ \\
\hline HbA1c, \% & $7.7(0.9)$ & $7.4(0.8)$ & $8.2(0.9)$ & $7.8(0.8)$ \\
\hline
\end{tabular}

Results are mean (standard deviation) unless otherwise indicated.

${ }^{a}$ Initial 24 adult subjects enrolled received $80 \%$ meal bolus; all subjects enrolled subsequently received $100 \%$ meal bolus.

${ }^{\mathrm{b}}$ Insulin dose averaged over the 7-day open-loop phase.

${ }^{\mathrm{c}}$ Insulin dose during the first 24-h HCL phase, including three meals and the overnight period.

HCL, hybrid closed loop. 


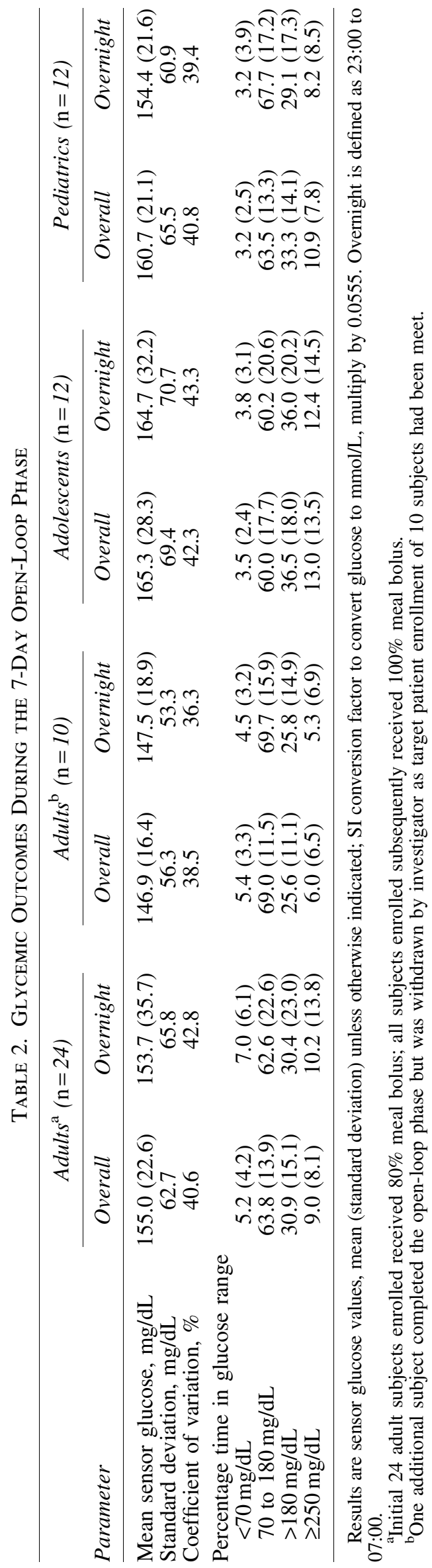

failures overall during the HCL phase and in these cases the Pods were replaced. Pod replacement for infusion site issues or temporary loss of Pod or CGM communication accounted for the interruption of closed loop.

\section{Hypo- and hyperglycemic treatments}

No serious adverse events were reported and there were no instances during the study in which the criteria were met to stop closed loop for prolonged hypo- or hyperglycemia. The overall hypoglycemia rate was 0.49 events $/ 24 \mathrm{~h}$ for the study overall, 0.25 events $/ 24 \mathrm{~h}$ in adults with $80 \%$ meal bolus, and $0.27,0.83$, and 0.83 events $/ 24 \mathrm{~h}$ in adults, adolescents, and pediatrics with $100 \%$ meal bolus, respectively. Overall, there were 45 treatments for hypoglycemic events, 15 events in each age group, in over $2088 \mathrm{~h}$ of HCL use. There were 16 treatments for hyperglycemia overall with $10(63 \%)$ events occurring in the adults receiving the $80 \%$ meal bolus.

\section{Discussion}

This multicenter, inpatient feasibility study demonstrated that the OmniPod personalized MPC algorithm performed well and was safe during day and night use over $36 \mathrm{~h}$ in adult, adolescent, and pediatric patients with type 1 diabetes. Positive glycemic outcomes were consistently observed across age groups that included adolescents who are typically challenged by insulin resistance and children as young as 6 years of age with enhanced insulin sensitivity.

A recent meta-analysis of 24 randomized controlled trials of closed-loop systems, including 585 patients, demonstrated that AP system use in the outpatient setting has resulted in $12 \%$ increased time in range $(70-180 \mathrm{mg} / \mathrm{dL})$ and a $50 \%$ reduction in the time spent in hypoglycemia compared to CSII with blinded CGM or SAP. ${ }^{6}$ Based on the outcomes of this meta-analysis, current HCL systems should achieve at least $70 \%$ of sensor glucose values between 70 and $180 \mathrm{mg} /$ $\mathrm{dL}$ with $<4 \%$ of values $<70 \mathrm{mg} / \mathrm{dL}$, a CV $<36 \%,{ }^{13}$ and a mean glucose of $\leq 155 \mathrm{mg} / \mathrm{dL}$, equivalent to an estimated A1c of $7.0 \% .^{14,15}$ The current study was able to meet all of these goals for adults, adolescents, and children, with the mean glucose within $2 \mathrm{mg} / \mathrm{dL}$ of the goal for each age group when a full meal bolus was given.

A significant benefit of AP systems in addition to overall improved glycemic control is the reduction in hypoglycemia in the overnight period. ${ }^{16,17}$ Positive overnight outcomes were also observed in the present study with $\sim 85 \%$ or greater percentage time spent in the target range $(70-180 \mathrm{mg} /$ dL) and $<0.7 \%$ time in hypoglycemia ( $<70 \mathrm{mg} / \mathrm{dL})$.

Limitations of this study include its short duration and that it was conducted in a supervised research center setting, with the systems managed by healthcare providers rather than the patients. A control arm was not included as this was the first feasibility study to determine the safety of the OmniPod HCL algorithm and to further refine the algorithm before commencing longer studies in a less supervised setting with protocols, including meal and exercise challenges.

The present study was conducted using a noncommercial investigational device. The OmniPod Horizon commercial system in development is intended to be differentiated from current HCL systems with its tubeless, on-body form factor, which includes the personalized MPC algorithm embedded in the Pod and communicating directly with the latest Dexcom 
Table 3. Glycemic Outcomes Over the 36-H Hybrid Closed-Loop Phase

\begin{tabular}{lcccc}
\hline Parameter & Adults $^{\mathrm{a}}(\mathrm{n}=24)$ & Adults $(\mathrm{n}=10)$ & Adolescents $(\mathrm{n}=12)$ & Pediatrics $(\mathrm{n}=12)$ \\
\hline Mean sensor glucose, mg/dL & $161.5(20.1)$ & $155.0(14.8)$ & $153.4(21.6)$ & $156.9(20.4)$ \\
Standard deviation, mg/dL & 54.0 & 46.2 & 48.6 & 53.3 \\
Coefficient of variation, \% & 33.4 & 29.8 & 31.8 & 34.0 \\
Percentage time in glucose range & & & & \\
$<54 \mathrm{mg} / \mathrm{dL}$ & $0.1(0.3)$ & $0.1(0.3)$ & $0.2(0.3)$ & $0.2(0.7)$ \\
$<60 \mathrm{mg} / \mathrm{dL}$ & $0.2(0.6)$ & $0.2(0.6)$ & $0.7(0.9)$ & $0.6(1.2)$ \\
$<70 \mathrm{mg} / \mathrm{dL}$ & $0.7(1.7)$ & $0.7(1.2)$ & $2.0(2.4)$ & $3.0(2.6)$ \\
$70 \mathrm{to} 140 \mathrm{mg} / \mathrm{dL}$ & $41.5(18.1)$ & $41.9(16.3)$ & $40.2(15.5)$ & $70.1(16.1)$ \\
70 to $180 \mathrm{mg} / \mathrm{dL}$ & $69.5(14.4)$ & $73.0(15.0)$ & $72.6(15.5)$ & $27.9(13.2)$ \\
$>180 \mathrm{mg} / \mathrm{dL}$ & $29.7(14.4)$ & $26.3(14.4)$ & $25.4(16.1)$ & $6.7(5.6)$ \\
$\geq 250 \mathrm{mg} / \mathrm{dL}$ & $8.0(7.5)$ & $3.6(3.7)$ & $4.9(6.3)$ & $1.0(2.2)$ \\
$\geq 300 \mathrm{mg} / \mathrm{dL}$ & $2.0(2.9)$ & $0.5(1.1)$ & $0.1(0.5)$ & \\
\hline
\end{tabular}

Results are sensor glucose values, mean (standard deviation) unless otherwise indicated; SI conversion factor to convert glucose to mmol/ L, multiply by 0.0555 .

${ }^{\mathrm{a}}$ Initial 24 adult subjects enrolled received $80 \%$ meal bolus; all subjects enrolled subsequently received $100 \%$ meal bolus.

Table 4. Glycemic Outcomes During the Overnight Hybrid Closed-Loop Phase

\begin{tabular}{lcccc}
\hline Parameter & Adults $^{\mathrm{a}}(\mathrm{n}=24)$ & Adults $(\mathrm{n}=10)$ & Adolescents $(\mathrm{n}=12)$ & Pediatrics $(\mathrm{n}=12)$ \\
\hline Mean glucose, mg/dL & $138.7(20.3)$ & $138.8(21.1)$ & $149.3(26.4)$ & $149.3(24.4)$ \\
Standard deviation, mg/dL & 34.7 & 29.9 & 34.6 & 35.2 \\
Coefficient of variation, \% & 25.1 & 21.6 & 23.2 & 23.6 \\
Percentage time in glucose range & & & & \\
$<54 \mathrm{mg} / \mathrm{dL}$ & $0.0(0.2)$ & $0.3(0.9)$ & $0.0(0.0)$ & $0.0(0.0)$ \\
$<60 \mathrm{mg} / \mathrm{dL}$ & $0.0(0.2)$ & $0.5(1.5)$ & $0.1(0.3)$ & $0.0(0.0)$ \\
$<70 \mathrm{mg} / \mathrm{dL}$ & $0.7(3.1)$ & $0.7(2.1)$ & $0.2(0.6)$ & $0.1(0.3)$ \\
$70 \mathrm{to} 140 \mathrm{mg} / \mathrm{dL}$ & $64.1(30.1)$ & $53.8(36.4)$ & $43.7(28.6)$ & $38.7(28.7)$ \\
70 to $180 \mathrm{mg} / \mathrm{dL}$ & $87.8(17.4)$ & $90.8(15.8)$ & $84.7(25.1)$ & $86.7(17.7)$ \\
$>180 \mathrm{mg} / \mathrm{dL}$ & $11.4(15.6)$ & $8.6(16.1)$ & $15.2(25.3)$ & $13.2(17.8)$ \\
$\geq 250 \mathrm{mg} / \mathrm{dL}$ & $1.0(4.7)$ & $0.6(1.9)$ & $1.5(4.9)$ & $2.2(5.8)$ \\
$\geq 300 \mathrm{mg} / \mathrm{dL}$ & $0.2(1.1)$ & $0.0(0.0)$ & $0.0(0.0)$ & $0.0(0.0)$ \\
\hline
\end{tabular}

Results are sensor glucose values, mean (standard deviation) unless otherwise indicated; SI conversion factor to convert glucose to mmol/ L, multiply by 0.0555 . Overnight is defined as 23:00 to 07:00.

${ }^{a}$ Initial 24 adult subjects enrolled received $80 \%$ meal bolus; all subjects enrolled subsequently received $100 \%$ meal bolus.

CGM system. The commercial product will also include a handheld device based on smartphone hardware using an Android operating system with advanced functionality, a state-of-the-art user interface, and cloud-based data management capabilities.

\section{Conclusions}

This feasibility study demonstrated that the OmniPod personalized MPC algorithm performed well and was safe during day and night use in adult, adolescent, and pediatric patients with type 1 diabetes. Longer term studies will assess the safety and performance of the algorithm under free living conditions with extended use in patients of all ages with type 1 diabetes.

\section{Acknowledgments}

The authors thank the study participants and their families and the dedicated staff at the participating research centers, including Liana Hsu and Ryan Kingman of the Stanford University Medical Center, Stanford, CA; Cari Berget, Laurel Messer, Katie Thivener, and Lindsey Towers of the Barbara Davis Center for Diabetes, University of Colorado
Denver, Denver, CO; Mei Mei Church, Wendy Bevier, Kelly Burke, and Tyler Jean of the Sansum Diabetes Research Institute, Santa Barbara, CA; and Margie Macarewich of the Diablo Clinical Research Center, Walnut Creek, CA. They appreciate the efforts of the research and development and clinical teams at Insulet Corporation, including Bonnie Dumais, Connor Gullifer, Stephanie Mandra, Daniel McCarthy, Shashi Murthy, Todd Vienneau, and Yibin Zheng, PhD. The authors thank Dr. Howard Zisser, of Verily Life Sciences, San Francisco, CA, and the University of California, Santa Barbara, Santa Barbara, CA, for his contributions to the study while he was an employee of Insulet Corporation. This work was funded by Insulet Corporation.

\section{Author Disclosure Statement}

Dr. Buckingham served as the principal investigator for the study and received research funding from Insulet Corporation, and has received research support from Bigfoot Biomedical, Dexcom, Medtronic, and Tandem. He is an advisory board member for Becton Dickinson, Novo Nordisk, and Sanofi. Dr. Forlenza-research funding from Animas, Bigfoot Biomedical, Dexcom, Insulet Corporation, Medtronic, Novo Nordisk, Tandem, and Type Zero, and is a 
speaker and advisory board member for Dexcom. Dr. Pinsker-research funding from Bigfoot Biomedical, Insulet Corporation, and Tandem, and has received product support for his institution from Animas, Dexcom, Insulet Corporation, LifeScan, and Roche. Dr. Wadwa-research funding from Bigfoot Biomedical, Dexcom, Insulet Corporation, Lexicon Pharmaceutical, Medtronic, Novo Nordisk, Tandem, and Xeris Pharmaceutical, and is an advisory board member for Eli Lilly \& Co and Novo Nordisk, and a consultant for MannKind. Dr. Christiansen-research funding from Abbott Diabetes Care, Dexcom, Insulet Corporation, and Medtronic. Dr. Dassau-patents related to the technology and royalty from Insulet Corporation and ModeAGC LLC and product support from Animas, Dexcom, Insulet Corporation, Roche, and Tandem. Dr. Schneider is a consultant to Insulet Corporation. Dr. Peyser is a consultant to Insulet Corporation and has consulted for Dexcom. Drs. Ly, Lee, and Layne and Mr. O'Connor are employees of Insulet Corporation.

\section{References}

1. Thabit H, Hovorka R: Coming of age: the artificial pancreas for type 1 diabetes. Diabetologia 2016;59:1795-1805.

2. Kowalski A: Pathway to artificial pancreas systems revisited: moving downstream. Diabetes Care 2015;38:10361043.

3. Voelker R: "Artificial Pancreas" is approved. JAMA 2016; 316:1957.

4. Bergenstal RM, Garg S, Weinzimer SA, et al.: Safety of a hybrid closed-loop insulin delivery system in patients with type 1 diabetes. JAMA 2016;316:1407-1408.

5. Garg SK, Weinzimer SA, Tamborlane WV, et al.: Glucose outcomes with the in-home use of a hybrid closed-loop insulin delivery system in adolescents and adults with type 1 diabetes. Diabetes Technol Ther 2017;19:155-163.

6. Weisman A, Bai JW, Cardinez M, et al.: Effect of artificial pancreas systems on glycaemic control in patients with type 1 diabetes: a systematic review and meta-analysis of outpatient randomised controlled trials. Lancet Diabetes Endocrinol 2017;5:501-512.

7. Clarke WL, Cox DJ, Gonder-Frederick LA, et al.: Reduced awareness of hypoglycemia in adults with IDDM. A prospective study of hypoglycemic frequency and associated symptoms. Diabetes Care 1995;18:517-522.
8. Dassau E, Zisser HC, Palerm, CC, et al.: Modular artificial beta-cell system: a prototype for clinical research. J Diabetes Sci Technol 2008;2:863-872.

9. Pinsker JE, Lee JB, Dassau E, et al.: Randomized crossover comparison of personalized MPC and PID control algorithms for the artificial pancreas. Diabetes Care 2016;39: 1135-1142.

10. Lee JB, Dassau E, Gondhalekar R, et al.: Enhanced model predictive control (eMPC) strategy for automated glucose control. Ind Eng Chem Res 2016;55:11857-11868.

11. Gondhalekar R, Dassau E, Doyle FJ, 3rd: Periodic zoneMPC with asymmetric costs for outpatient-ready safety of an artificial pancreas to treat type 1 diabetes. Automatica 2016;71:237-246.

12. Maahs DM, Buckingham BA, Castle JR, et al.: Outcome measures for artificial pancreas clinical trials: a consensus report. Diabetes Care 2016;39:1175-1179.

13. Monnier L, Colette C, Wojtusciszyn A, et al.: Toward defining the threshold between low and high glucose variability in diabetes. Diabetes Care 2017;40:832-838.

14. Jaeb Center for Health Research-HbA1c Estimator: www. jaeb.org/a1c (accessed August 30, 2017).

15. Beck RW, Connor CG, Mullen DM, et al.: The fallacy of average: how using $\mathrm{HbA} 1 \mathrm{c}$ alone to assess glycemic control can be misleading. Diabetes Care 2017;40:994-999.

16. Thabit H, Elleri D, Leelarathna L, et al.: Unsupervised overnight closed loop insulin delivery during free living: analysis of randomised cross-over home studies in adults and adolescents with type 1 diabetes. Lancet 2015;385 Suppl 1:S96.

17. Ly TT, Breton MD, Keith-Hynes P, et al.: Overnight glucose control with an automated, unified safety system in children and adolescents with type 1 diabetes at diabetes camp. Diabetes Care 2014;37:2310-2316.

Address correspondence to: Bruce A. Buckingham, MD Division of Endocrinology Department of Pediatrics Stanford School of Medicine Stanford, $C A$ Mailing address: 780 Welch Road Palo Alto, CA 94305

E-mail: buckingham@stanford.edu 